\title{
Elitismo na literatura sobre a produtividade dos autores
}

\section{Rubén Urbizagástegui Alvarado}

Mestre em ciência da informação pelo Instituto Brasileiro de Informação em Ciência e Tecnologia da Universidade Federal de Rio de Janeiro (IBICT/UFRJ). Mestre em biblioteconomia pela Universidade de Pittsburgh (Pensilvania, Estados Unidos). Pós-graduação em sociologia e comunicação social na Universidade de Brasília (UnB). Doutor em ciência da informação pela Universidade Federal de Minas Gerais (UFMG)

E-mail: ruben@ucr.edu

\section{Resumo}

O objetivo deste artigo é identificar, descrever e analisar os autores que conformam a elite numa subárea do campo da bibliometria: a produtividade dos autores, conhecida também como a Lei de Lotka. Como unidades de análises foi tomado cada um dos 376 diferentes autores produtores dos 390 artigos, capítulos de livros e trabalhos apresentados em congressos sobre a chamada Lei de Lotka, desde 1922 até 2003. Encontrou-se que a elite está formada por 17 autores que participaram da produção de cinco ou mais documentos cada um e contribuindo com pouco mais de uma terceira parte dos artigos.

\section{Palavras-chave}

Lei do elitismo. Lei de Price. Lei de Lotka. Produtividade dos autores. Cienciometria. Bibliometria. Informetria.

\section{Choice in the literature about productivity of authors}

\begin{abstract}
The objective of this article is to identify and analyze the authors who make up the elite in a sub-area of the bibliometric field: the author's productivity is also known as the "Lotka's law". The units of analysis were taken from 376 different authors producing of 390 articles, book chapters, and works presented in congresses, from 1922 up to 2003. The conclusion is that the elite was made up by 17 authors who had each one participated at least in the production of five or more documents totalizing a contribution of over a third of all documents.
\end{abstract}

\section{Keywords}

Elitism. Price's law. Lotka's law. Authors' productivity. Scientometrics. Bibliometrics. Informetrics. 


\section{INTRODUÇÃO}

O objetivo desta pesquisa é identificar, descrever e analisar os autores que conformam a elite numa subárea do campo da bibliometria: a produtividade dos autores, conhecida também como a Lei de Lotka. Se a publicação de documentos segue um padrão tradicional no processo de comunicação da ciência, essa identificação e descrição da elite podem ser realizadas via análise da literatura produzida sobre o assunto. Portanto, os documentos publicados poderiam constituir também confiável forma de estudar esta subárea científica do campo da bibliometría.

A distribuição da produtividade dos autores numa coordenada cartesiana é uma distribuição tão inclinada, que inspirou Price (1963) a propor a Lei do Elitismo. Segundo esta lei, se k representa o número total de contribuintes numa disciplina, $\sqrt{k}$ representaria a elite da área estudada, assim como o número de contribuintes que gera a metade de todas as contribuições. Este princípio parece se aplicar tanto às artes como às ciências e "pronto se fez notório que o fenômeno primeiro observado por Lotka é intrínseco à natureza do processo criativo na ciência" (VALCHY, 1972). Por exemplo, Moles (1958, 1966) observou este fenômeno no campo da música, no qual 16 compositores acumulam a metade de todas as execuções de música clássica. A essa característica da produtivi-dade dos autores se tem chamado também de "teoria da raiz quadrada" ou Lei de Price. Esta lei estabelece que a raiz quadrada de todos os autores produtores de literatura em determinado campo produzirá, quando menos, a metade de todos os artigos publicados pela população de autores estudados. Especificamente, Price sugere que,

Ao se contar a produção total daqueles que produzem $n$ artigos, parece que o grande número de pequenos produtores contribuem tanto quanto o total do pequeno número dos grandes produtores; num simples caso esquemático, se pode mostrar uma simetria no ponto correspondente à raiz quadrada do número total de pessoas, ou as contribuições dos grandes produtores. Se existem 100 autores e se o mais prolífico produz 100 artigos, a metade de todos os artigos terá sido escrita pelos 10 mais prolíficos autores, e a outra metade por aqueles com menos de 10 artigos cada um. De fato, neste caso ideal, um quarto dos artigos terão sido escritos pelas duas pessoas mais produtivas, e outro quarto por aqueles que publicaram somente um ou dois itens (PRICE, 1963, p. 46).

E insiste que isso produziria um método objetivo para separar os maiores dos menores contribuintes, em outras palavras, separar o joio do trigo, tanto que

pode-se estabelecer um limite e dizer que a metade do trabalho é feito por aqueles com mais de 10 artigos, ou que o número dos grandes produtores parece ser da mesma ordem de magnitude que a raiz quadrada do número total de autores (PRICE, 1963, p. 46).

Portanto, fazendo-se os cálculos, pode-se chegar à conclusão de que, independentemente do tamanho da população estudada,

aproximadamente $75 \%$ daqueles que escrevem um só artigo nunca mais voltam a escrever; e que $10 \%$ dos escritores altamente prolíficos são os que produzem aproximadamente a metade da literatura científica mundial (PRICE, 1963, p. 37).

A metade dos artigos científicos do mundo é escrita por aqueles que escrevem mais de vinte artigos em toda sua vida, e o número desses autores altamente produtivos é aproximadamente a raiz quadrada do total dos autores (PRICE, 1963, p. 41).

Essas insistentes afirmações deram lugar a polêmicas, uma das quais terminou com um artigo conjunto dos envolvidos nessa polêmica questionando a consistência da "teoria da raiz quadrada" ou Lei de Price. Os autores se focaram, consistentemente, nas consequências matemáticas da Lei de Lotka e nas conexões matemáticas entre a Lei de Lotka e 
a Lei de Price, e não no ajuste a dados empíricos sobre a produtividade dos autores para terminar afirmando que

a validade da Lei de Price não depende necessariamente da validade da Lei de Lotka, e que somente pode ser julgada sobre a base de evidências empíricas (ALLISON et al., 1976, p. 274).

Essas evidências empíricas foram procuradas por Coile (1977), com dados procedentes de 15 áreas diferentes, tendo o grupo de elite nesses campos produzido uma proporção que ia de $9 \%$ (entomologia) a 38\% (matemática) dos produtores, sugerindo que são necessárias mais pesquisas sobre a questão da produtividade do grupo de elite, já que os autores mais prolíficos estimados como a raiz quadrada da população estudada não parece produzir a metade dos artigos. Adicionalmente, afirma que dados sobre a produtividade científica de físicos, matemáticos, biólogos, cientistas da computação, econometristas, pesquisadores operacionais e entomólogos sugerem que a raiz quadrada do total dos autores produz, em média, $25 \%$ do total dos artigos (COILE, 1977).

Também Gupta e colaboradores (1996), estudando a produtividade dos autores na pesquisa da genética da batata, observaram que a elite dos autores calculados como a raiz quadrada da população estudada produziu somente $26.12 \%$ do total de artigos publicados, muito abaixo dos $50 \%$ sugerido por Price. Identicamente, Gupta e Karisiddappa (1996), estudando a produtividade dos autores no campo da genética, observaram que a produção da elite dos autores calculados como a raiz quadrada da população estudada variava entre $40.41 \%$ e $42.93 \%$, ainda abaixo dos 50\% sugeridos por Price.

Esse mesmo padrão tem sido observado no campo da psicologia do esporte, no qual "aproximadamente $24 \%$ de todos os artigos publicados nos periódicos de psicologia do deporte foram produzidos por $3 \%$ dos pesquisadores publicando ativamente. Ainda mais, $10 \%$ dos pesquisadores foram responsáveis

Ci. Inf., Brasília, v. 38, n. 2, p. 69-79, maio/ago. 2009 por aproximadamente $44 \%$ de todos os artigos produzidos" (BAKER; ROBERTSON-WILSON; SEDGWICK, 2003, p. 481). Também Berg e Wagner-Döbler (1996) não encontraram esses $50 \%$ de produtividade da elite no campo da lógica matemática. Similarmente, Gupta, Sharma e Kumar (1998), estudando o campo da física na Índia, encontraram que a contribuição da raiz quadrada dos autores variava nos diferentes períodos estudados com um máximo de $44.25 \%$ para o período de $1800-1890$ e um mínimo de $13.08 \%$ para o período 1900-1950, sendo a média dessa produtividade $37.59 \%$, novamente abaixo do esperado pela lei de elitismo de Price.

Não obstante, nenhum trabalho tem sido desenvolvido estudando um campo quase homogêneo e sem divisões internas como a que oferece a produtividade dos autores, conhecida também como a Lei de Lotka. Existe essa elite nos estudos da produtividade de autores? E, se existe, que autores a constituem? Essa elite de autores identificados é responsável pela metade da literatura produzida nesta área? Esta pesquisa pretende responder a estas interrogações.

\section{METODOLOGIA}

Este trabalho é empreendido como um intento de identificar os autores mais produtivos ou a elite dos autores produtores da literatura sobre a Lei de Lotka. Por essa razão, como unidades de análises foi tomado cada um dos autores produtores dos artigos, capítulos de livros e trabalhos apresentados em congressos sobre a chamada Lei de Lotka, desde 1922 até 2003, isto é, um longo período de 82 anos. Para identificar os autores contribuindo com artigos nesta área, foi feita uma busca usando a estratégia Dial Index em todas as bases de dados de DIALOG, com os termos Lotka?(5n)Law? encontrando-se 50 bases de dados que pelo menos continham um artigo sobre o assunto pesquisado. Esta estratégia de busca produziu um total de 315 registros, que, depois da depuração dos duplicados e falsas recuperações, foram acumulados num total de 275 referências bibliográficas. Essas referências foram depois trasladadas ao PROCITE 5.0 para a 
elaboração de uma base de dados específica sobre o assunto. Posteriormente, foi realizada minuciosa leitura de cada um dos artigos identificados na busca, dedicando especial atenção a cada citação efetuada no documento lido. Depois, cada citação referente à Lei de Lotka era confrontada com a base de dados e nela incorporada, se não tivesse sido identificada na busca anterior no DIALOG. Também foram realizadas buscas no Information Science Abstract (ISA), Library Literature (LL) e Library and Information Science Abstract (LISA). Com esta leitura minuciosa, foi produzida uma bibliografia analítica da Lei de Lotka que lista um total de 390 referências bibliográficas contendo artigos de periódicos, monografias, capítulos de livros, comunicações em congressos, literatura gris, cartas dirigidas a editores de revistas especializadas em biblioteconomia e ciência da informação (URBIZAGÁSTEGUI ALVARADO, 2005). Esta bibliografia analítica de 390 referências produzidas entre 1922 e 2003 constitui o universo desta pesquisa.
O período coberto pela literatura recuperada é suficientemente longo para assegurar a publicação de artigos sobre este assunto nos periódicos do campo da ciência da informação e afins.

Para a contagem dos autores produtores de artigos optamos pelo sistema de contagem direta, completa e ajustada. Isto significa, ainda, que os múltiplos autores de um único artigo serão contados e atribuídos como autores contribuintes à produção de cada artigo ou documento identificado no levantamento bibliográfico. Para a mensuração dos dados, consideramos o modelo proposto por Price (1963) $\sqrt{k}$, isto é, a raiz quadrada do total da população estudada.

\section{RESULTADOS}

No período estudado foram identificados 390 documentos produzidos por 376 autores diferentes. Uma classificação, segundo os tipos de documentos produzidos, pode ser observada na figura 1. Os

\section{FIGURA 1}

\section{Tipo de documentos produzidos}

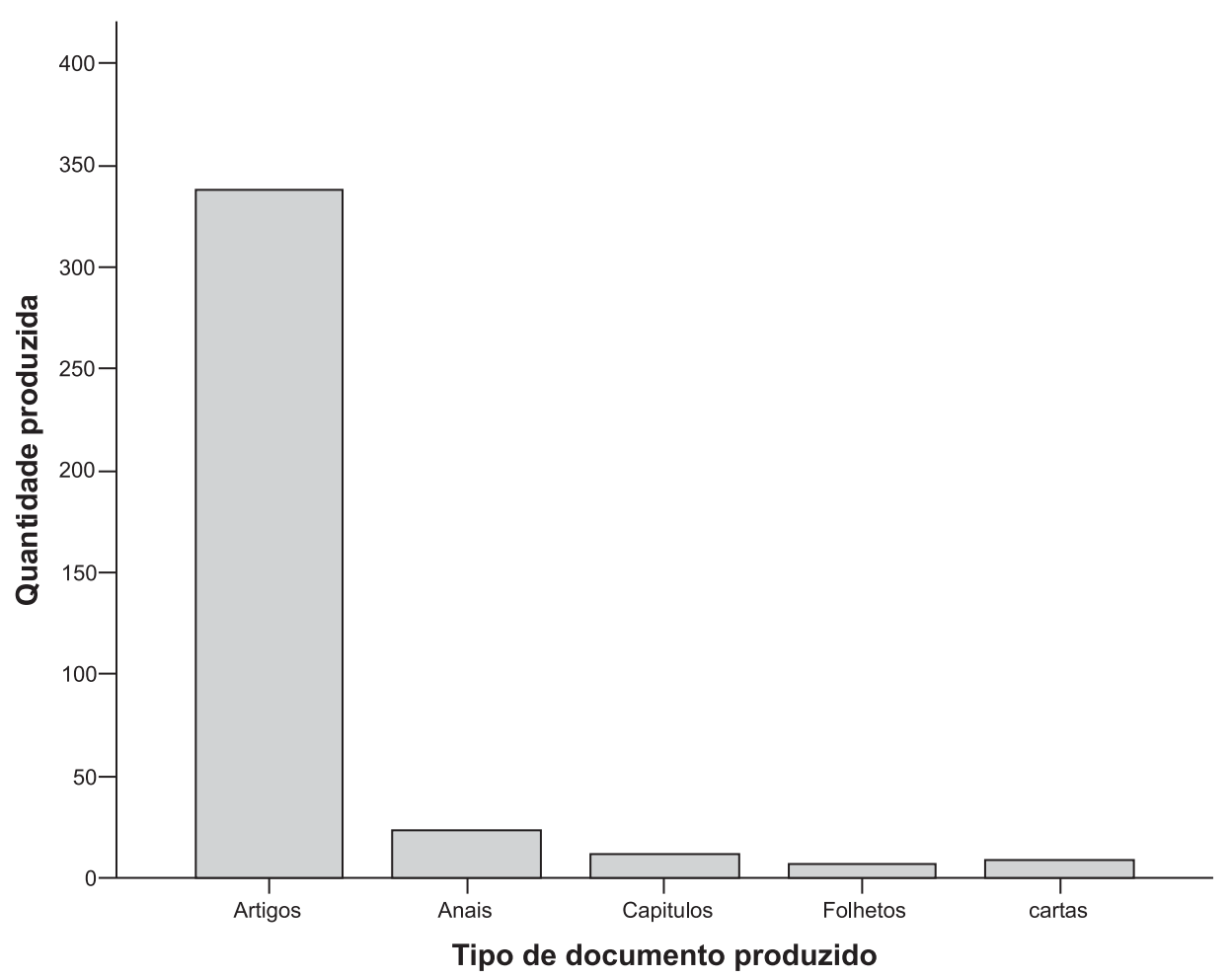


autores produtores de literatura sobre este assunto têm preferência por apresentar suas contribuições na forma de artigos publicados nos periódicos do campo da ciência da informação; $86.7 \%$ dessas contribuições têm esta forma de publicação, deixando apenas um espaço de 13\% para os outros tipos de contribuições. Nesse pequeno espaço, as contribuições apresentadas em anais de congressos $(6.2 \%)$ ocupam um segundo estrato pouco significativo, assim como as publicações em capítulos de livros $(3.1 \%)$, em folhetos $(1.8 \%)$ e as cartas enviadas aos editores desses periódicos atinge apenas $2.3 \%$ dessas contribuições.

Estes resultados parecem naturais em termos da prática cientifica, afinal periódicos (eletrônicos ou não) continuam sendo o canal de comunicação formal por excelência para os cientistas e onde os artigos apresentam os resultados de pesquisa corrente na íntegra ou ainda resultados parciais. Já os livros ou capítulos de livros representam conhecimento consolidado e cristalizado, mas que na produção sobre a lei de Lotka tem uma baixa percentagem $(3.1 \%)$ de presença. Dai que pesquisas

\section{FIGURA 2}

Documentos produzidos segundo os idiomas nesta subárea da bibliometria estejam em pleno desenvolvimento.

A figura 2 mostra as contribuições classificadas segundo os idiomas de publicação. Pode-se observar a hegemonia do inglês, com 310 (79.5\%) das contribuições publicadas neste idioma, deixando apenas um espaço de $20.5 \%$ para ser compartidos pelos outros idiomas. Neste aspecto, as publicações no idioma espanhol ocupam um segundo estrato, com $51(13.1 \%)$ das contribuições, seguido do português, com 16 (4.1\%), e assim sucessivamente o idioma alemão $4(1 \%)$, chinês $4(1 \%)$, turco, servocroata e russo, com apenas $1(0.3 \%)$ dos trabalhos produzidos. Isto também não é nada surpreendente. Já é conhecida a hegemonia do idioma inglês na comunicação cientifica, tanto que, segundo Price (1971, p. 257), o inglês constitui pouco mais da metade da produção filosófica e científica do mundo, embora os cientistas não leiam muito e virtualmente nada em idiomas estrangeiros. Para ser mais precisos, “... olham a literatura estrangeira através de [seus] óculos obscuros que deixam ver somente uma décima parte daquilo que realmente existe" (PRICE, 1971, p. 258).

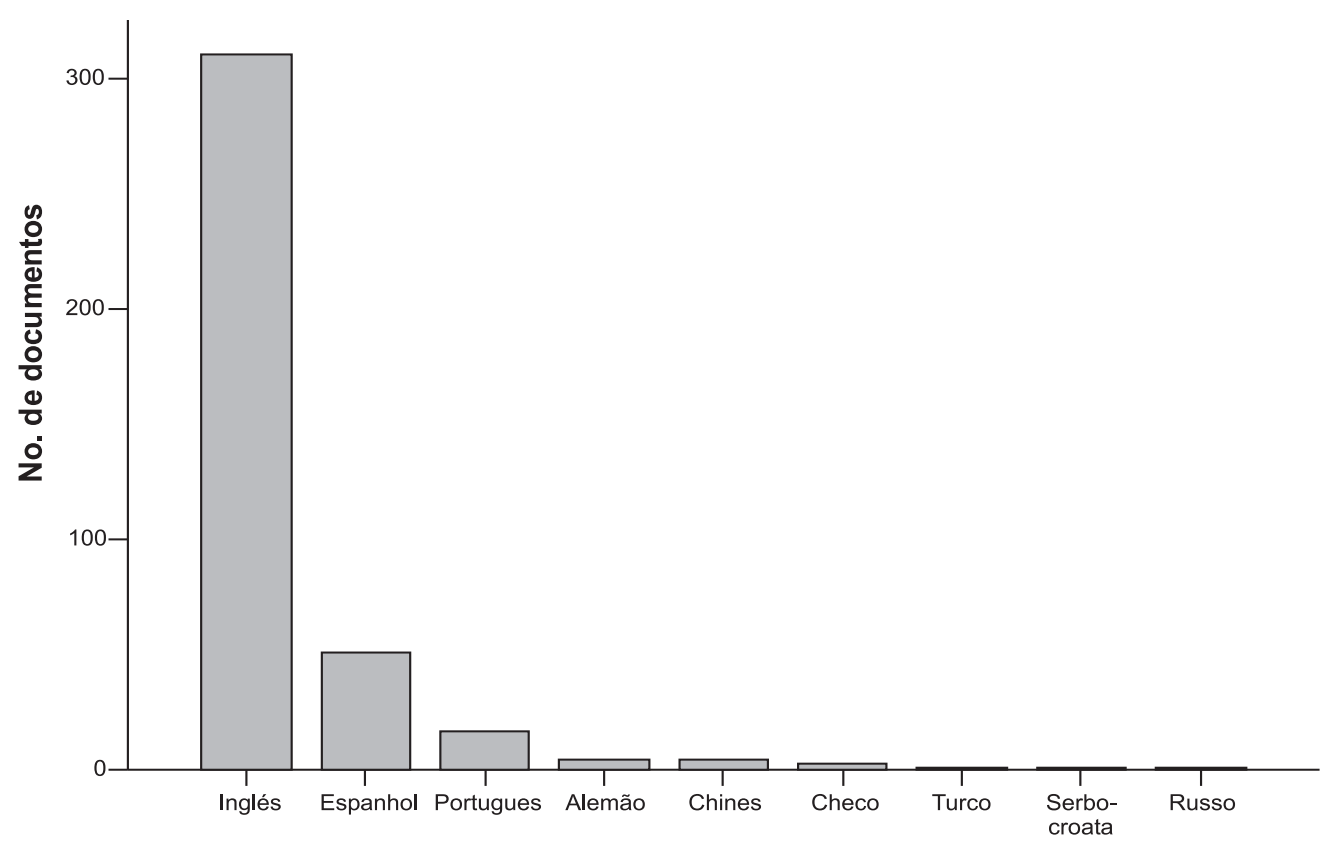

Idiomas 
Quando se credita a produtividade de autores somente aos autores principais e se ignoram os autores colaboradores (contagem direta), no período estudado foi levantado um total de 229 autores que, em conjunto, produziram 381 trabalhos, já que nove documentos que foram cartas dirigidas aos editores de publicações acadêmicas foram retirados do universo de analise. Desse total de autores, 77.3\% deles participaram na produção de um único artigo, mas em conjunto produziram somente $46.5 \%$ dos artigos, com uma produção média de 1.0 artigo por autor. Porém, a produtividade média do total de autores é de 1.66 artigos por autor, com uma variância de 3.3 artigos, um desvio padrão de 1.82 artigos e um índice de dispersão igual a 1.97 artigos.

Com a forma de contagem completa, quando se credita a produtividade a todos os autores, independentemente de se eles participaram da produção do trabalho como autores principais ou colaboradores, foram observados 376 autores, sendo $76.86 \%$ deles participantes na produção de um único artigo, mas em conjunto este grupo de produtores únicos foi responsável somente por $40 \%$ dos trabalhos produzidos. Nessa faixa de autores com um único artigo, a média é de apenas 0.5 artigos por autor. Porém, a produtividade média geral encontrada foi de apenas de 1 artigo por autor, com uma variância de 4.84 artigos, um desvio padrão de 2.2 artigos e um índice de dispersão igual a 4.77 artigos.

Com a contagem ajustada ou fracionada, quando cada um dos autores principais e secundários são creditados com uma fração ou porção da contribuição total de artigos, foi identificado que $84 \%$ deles participaram na produção de 0.5 (médio) artigo, mas em conjunto produziram $45 \%$ dos trabalhos. Para este caso, os dados das contribuições tiveram de ser agrupados em intervalos de classe. A produtividade média geral continua sendo de apenas um artigo por autor, com uma variância de 2.0131 artigos, um desvio padrão de 1.4188 artigos, e um índice de dispersão igual a 2.2134 artigos por autor. A tabela 1 mostra a demografia desta distribuição de frequências, segundo a forma de contagem.
TABELA 1

Demografia segundo a forma de contagem

\begin{tabular}{lrrr}
\hline Parâmetros & $\begin{array}{r}\text { Contagem } \\
\text { direta }\end{array}$ & $\begin{array}{r}\text { Contagem } \\
\text { completa }\end{array}$ & $\begin{array}{r}\text { Contagem } \\
\text { fracionada }\end{array}$ \\
\hline Media & 1.6710 & 1.0133 & 0.9095 \\
Variância & 3.2966 & 4.8372 & 2.0131 \\
Desvio padrão & 1.8157 & 2.1994 & 1.4188 \\
Índice de dispersão & 1.9727 & 4.7737 & 2.2134 \\
Grupo de elite & 15.13 & 19.391 & 19.391 \\
\hline
\end{tabular}

Para a contagem direta, a variância é duas vezes maior do que sua média. Para a contagem completa, a variância é quase cinco vezes maior do que a média. Para a contagem ajustada ou fracionada, a variância é duas vezes maior do que a média. Esses valores indicam superdispersão no padrão do comportamento produtivo dos autores. Essa apreciação é confirmada pelo índice de dispersão da distribuição, pois esse valor é maior do que a média, significando que os autores trabalham de forma isolada e sem maiores contatos com os pares, porém seguindo com atenção a produtividade da área. Em outras palavras, esses autores preferem trabalhar individual e isoladamente.

Este mesmo fenômeno já havia sido observado por McCreery e Pao (1984) no campo da etnomusicología. Estes autores afirmam que os cientistas sociais e humanistas em geral preferem trabalhar individualmente, e não em colaboração. Se considerarmos a ciência da informação e em especial a bibliometria como uma área afim das ciências sociais, talvez isso seja também o caso da produtividade de autores relacionados com a Lei de Lotka.

Para os autores produtores de trabalhos sobre a Lei de Lotka, quando se considera somente a contagem direta, estimou-se uma elite de 15.13 autores (arredondado a 15 autores), mas, quando se considera a contagem completa e ajustada, estimou-se uma elite de 19.39 autores (arredondado a 19 autores), porém a cifra mais próxima a essa quantidade é de 17 autores, portanto, nesta pesquisa, considerou-se que a elite é formada por aqueles 17 
autores que participaram da produção de cinco ou mais artigos cada um e contribuindo com pouco mais de uma terceira parte (37.8\%) dos artigos. Esses autores, arranjados segundo suas produtividades, estão listados na tabela 2 e atingem apenas 4.5\% da população estudada, sugerindo, assim, que este é uma subárea da bibliometria muito jovem e em pleno desenvolvimento. Quantidades similares já tinham sido observadas por Dresden (1922) no campo das matemáticas, onde mais ou menos 5\% dos autores contribuíam com 30\% do total de artigos estudados. Esses resultados, porém, estão muito longe das propostas de Price (1963), no sentido de que aproximadamente 10\% dos autores mais produtivos são responsáveis pela metade da literatura gerada.

Como se pode observar na figura 3, no caso da literatura produzida sobre a Lei de Lotka, aproximadamente $25 \%$ dos trabalhos foram produzidos por $5 \%$ dos autores, bem como $50 \%$ dessa literatura foram produzidos por aproximadamente 20\% dos autores. Pode-se observar, também, que
TABELA 2

Grupo de elite dos autores produtores de literatura sobre a Lei de Lotka

$\mathbf{N}^{\circ}$ de trabalhos

Autores produzidos

Ronald Rousseau 18

Leo Egghe 16

John C. Huber 10

B. M. Gupta 9

Jan Vlachy 9

Roland Wagner-Dobler 9

Abraham Bookstein 8

Davendra K. Gupta 8

Miranda Lee Pao 8

Andras Schubert 8

Rubén Urbizagástegui 8

Kee H. Chung 6

Suresh Kumar 6

Paul Travis Nicholls 6

Russell C. Coile 5

Raymond A. K. Cox 5

V. L. Kalyane 5

FIGURA 3

Curva de concentração dos trabalhos versus autores

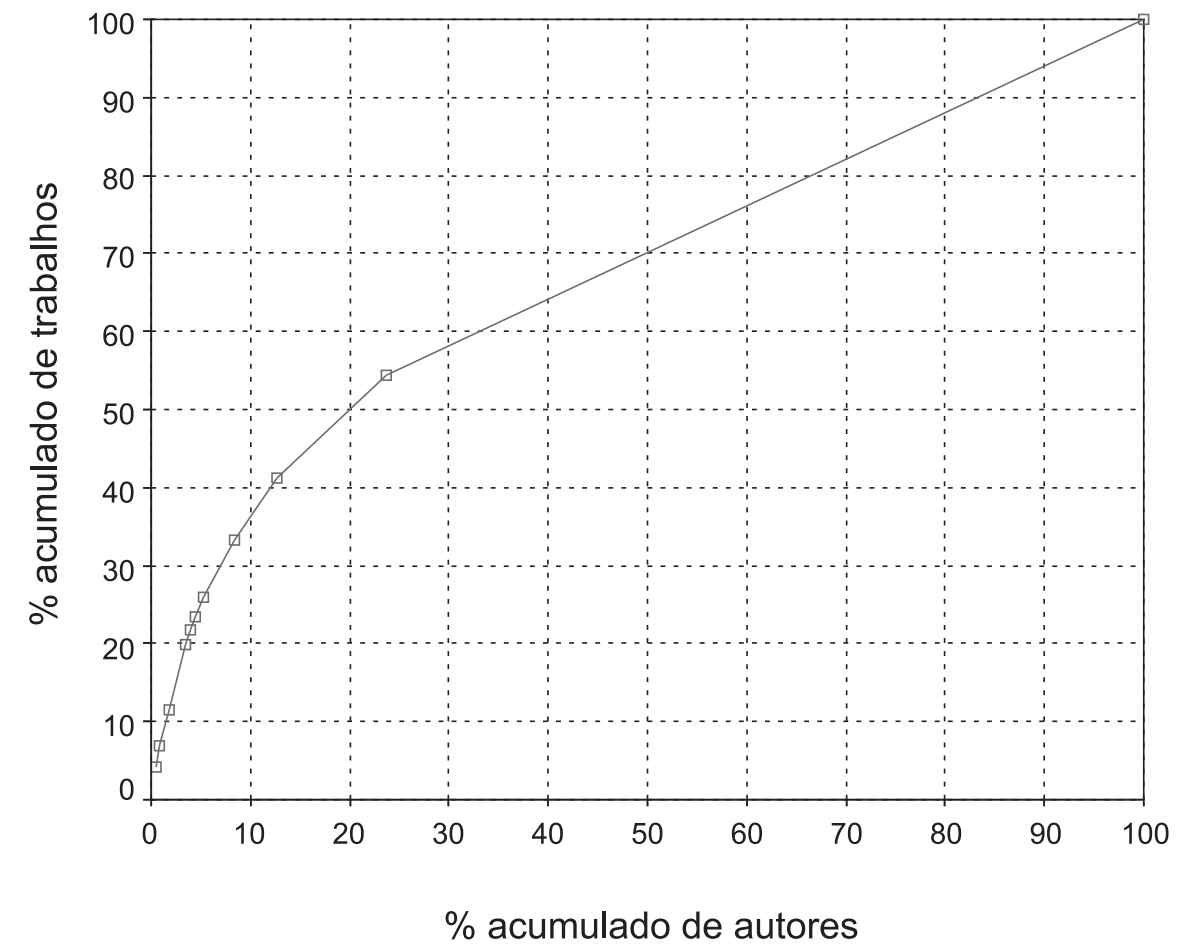

Ci. Inf., Brasília, v. 38, n. 2, p. 69-79, maio/ago. 2009 
$10 \%$ dos autores produziram aproximadamente $35 \%$ das contribuições. Portanto, essas são evidências suficientes para afirmar que, embora pequeno núcleo de autores tenha colaborado regularmente neste campo, a maior parte deles ainda é de iniciantes na pesquisa deste assunto. Não obstante, é oportuno esclarecer que o grupo de elite não é responsável pela metade da literatura produzida. Quantidades similares já haviam sido observadas por Carpintero; Pieró; Quintanilla (1977), que verificaram que 10\% dos autores mais produtivos foram responsáveis por $36 \%$ do total dos trabalhos publicados no periódico Anuário de Psicologia, pesquisado no período de 1969 a 1974. Igualmente, Nagpal e colaboradores (1966) observaram resultados quase similares no campo da engenharia eletrônica, tendo 25\% do total de artigos sido produzidos por $19 \%$ dos autores.

Similarmente, Rahman e Malik (1966) constataram comportamento similar na literatura de botânica, sendo $50 \%$ da literatura produzido por $11.5 \%$ dos autores. Também na literatura de finanças (CHUNG; COX, 1990, p. 308) essas projeções não se cumprem. Portanto, a expectativa de generalização desta proposta parece estar errada, já que, segundo Vlachy (1974),

não existem fortes evidências para sustentar que a produtividade de pesquisas ou o volume das comunicações escritas sejam os critérios que definam o pertencimento à elite de um grupo de pesquisa. A elite de um campo de pesquisa necessita ser mais bem definida, já que seus membros têm interesses profissionais menos difusos que os outros membros menos produtivos ou externos a essa elite. Isso pode significar que o fato de pertencer a uma elite pode estar caracterizado por uma maior especialização ou interesse em linhas de pesquisa específicas dentro de poucas subdisciplinas.

Essa afirmação parece ser confirmada por esta pesquisa.
Também existe o questionamento de que essa teoria da raiz quadrada ou lei de Price tem sido acriticamente aceita e que na bibliometria e cienciometria lhe tem sido outorgado um status de lei sem ter sido sujeita a um teste de validade (NICHOLLS, 1988, p. 469). Essa crítica é endossada por Berg e Wagner-Döbler (1996, p. 345) quando afirmam que "a lei da raiz quadrada de Price certamente é uma conjetura inadequada para as distribuições empíricas do tipo de Lotka”. Como essa Lei foi estabelecida verbalmente, recentemente, Glanzel e Schubert (1985) formularam uma definição formal mais exata da lei. Esses autores adotaram uma abordagem da forma ranking da frequência, mas Nicholls (1988) proporciona uma abordagem pelo tamanho-dafrequência, que corresponde, mais acuradamente, à representação da Lei de Lotka.

Em contrapartida, para responder às duvidas de Cattell (1910), no sentido de que não se sabia se o progresso na ciência se devia a um grande número de persistentes trabalhadores ou ao gênio de poucos autores, Dennis (1955) estudou as bibliografias produzidas nos campos da música secular, gerontologia e geriatria, geologia norteamericana, paralisia infantil, química, linguística, assim como os livros representados nos catálogos da Biblioteca do Congresso Americano. O autor concluiu sua pesquisa afirmando que "havia mostrado claramente que a maioria das contribuições criativas eram feitas por uma minoria dos contribuintes". Isso não significa ignorar que os autores de baixa produtividade não tenham nenhum impacto na produtividade dos autores altamente produtivos. De fato, como bem afirma Lolas (1986), "O acesso à publicação, longe de ser um jogo democrático, está marcado por desigualdades básicas". Sabemos que a produtividade dos autores também está marcada pela posse ou não de capitais culturais e também de capitais sociais e até políticos, tanto quanto pela posição ocupada por esses autores no campo hierarquizado da produtividade científica (BOURDIEU, 1973). Infelizmente, os aspectos sociais da produtividade científica ainda não têm sido suficientemente explorados na biblioteconomia 
TABELA 3

Categorização estratificada da produtividade dos autores

\begin{tabular}{lccccc}
\hline $\begin{array}{l}\mathbf{N}^{\circ} \text { de } \\
\text { Categoria }\end{array}$ & $\begin{array}{c}\% \text { de } \\
\text { Autores }\end{array}$ & $\begin{array}{c}\mathbf{N}^{\circ} \text { de } \\
\text { Autores }\end{array}$ & $\begin{array}{c}\% \text { de } \\
\text { trabalhos }\end{array}$ & $\begin{array}{c}\text { Produtividade } \\
\text { trabalhos }\end{array}$ & Média \\
\hline $\begin{array}{l}\text { Grandes produtores } \\
\text { (10 ou mais trabalhos) }\end{array}$ & 3 & 0.8 & 35 & 9.2 & 11.7 \\
$\begin{array}{l}\text { Produtores moderados } \\
(5 \text { a } 9 \text { trabalhos) }\end{array}$ & 14 & 3.7 & 78 & 20.5 & 5.6 \\
$\begin{array}{l}\text { Aspirantes } \\
(3 \text { a } 4 \text { trabalhos })\end{array}$ & 25 & 6.6 & 61 & 16.0 & 2.4 \\
$\begin{array}{l}\text { Transeuntes } \\
(1 \text { a } 2 \text { trabalhos })\end{array}$ & 334 & 88.8 & 207 & 54.3 & 0.6 \\
Total & 376 & 100.0 & 381 & 100.0 & 1.01 \\
\hline
\end{tabular}

e ciência da informação, embora Bourdieu ${ }^{1}$ tenha produzido uma valiosa e vasta literatura a esse respeito. Não obstante, Price (1986, p. 220), analisando a distribuição da produtividade dos autores, categoriza os componentes de uma comunidade cientifica em:

a) transientes ou transeuntes, definidos como aqueles que publicam somente um artigo científico durante determinado ano;

b) recrutas, aqueles que começam a publicar em determinado ano e se juntam à população dos continuantes;

c) terminantes, aqueles que finalizam sua atividade de publicação durante determinado ano e deixam a população dos continuantes;

d) núcleo dos continuantes, aqueles que publicam em determinado ano e continuam sua atividade de publicação a cada ano, ainda por um longo período.

e) continuantes, mas não pertencentes ao núcleo dos continuantes, aqueles que publicam num ano, mas têm dois terços de probabilidades de continuar publicando ocasionalmente por um longo período.

1 Uma vasta bibliografia sobre sua produtividade incluindo o
assunto em questão pode ser consultada em Urbizagástegui Alvarado Ci. Inf., Brasília, v. 38, n. 2, p. 69-79, maio/ago. 2009 f) continuantes não publicantes, aqueles que também têm dois terços de probabilidade de publicar trabalhos científicos em determinado ano.

As propriedades demográficas dos transientes e continuantes estão associadas, respectivamente, às baixas e altas taxas de produtividade científica. Os transientes publicam somente uma vez. Os continuantes têm média de produtividade de dois artigos por ano. Essas características demonstrariam que a estrutura e crescimento de uma comunidade científica é hiperbólica. Mais, ainda, demonstraria a necessidade de se conhecer a dinâmica do processo que governa a emergência, sobrevivência e mortalidade dos elementos que compõem uma comunidade de produtores científicos. Portanto, para ressaltar ainda mais a produção estratificada dos autores, conforme se pode verificar na tabela 3, foi organizado um esquema de categorização em quatro níveis bem diferenciados.

Observa-se a alta taxa de transeuntes e aspirantes que, em conjunto, atingem $95.5 \%$ da população estudada, comparada ao grupo de $4.5 \%$ dos moderados e grandes produtores que conformam a elite dos autores produtores da literatura sobre a Lei de Lotka. Observa-se, também, a estratificação da produtividade média nas diferentes categorias. Esta diferença quase se multiplica por quatro, à medida que se ascende das categorias de transeuntes 
a aspirantes (taxa de 1 a 4), no entanto esta taxa se duplica (taxa de 1 a 2 ) nas categorias dos aspirantes a produtores moderados e dos produtores moderados para os grandes produtores.

Aliás, uma estratificação semelhante tem sido observada, no campo da psicologia, por Carpintero, Pieró e Quintanilla (1977) e, na geologia chilena, por Urbizagástegui e Cortés (2002). Como se sabe, para aqueles autores que estão trabalhando na frente de pesquisa, a publicação não é um simples indicador, senão também o produto final de seu esforço criativo. Portanto, existe alta correlação entre a qualidade e a quantidade das publicações, em razão de que o êxito na publicação renova os esforços para mais publicações. Ao contrário, a rejeição dos manuscritos submetidos a um periódico acadêmico tende a inibir os esforços por publicar novamente.

\section{CONCLUSÕES}

Esta pesquisa demonstrou que, no período estudado, a média geral da produtividade dos autores da literatura sobre a Lei de Lotka foi de 1.7, 1.0 e 0.9 trabalho por autor, segundo a forma de contagem direta, completa e fracionada. Esta média é muito similar à média de 0.89 trabalho por autor reportados por Rivas e Peiró (1981), no campo da psicometria, e está próxima à média de 1.83 trabalho por autor, verificado por McCreery e Pao (1984), no campo da etnomusicología.

Identificou-se também uma elite de 17 autores que atingiram a média de 8.2 trabalhos por autor e produziram pouco mais de um terço $(37.8 \%)$ da literatura publicada. Esta quantidade é similar á encontrada por Carpintero, Pieró e Quintanilla (1977), na revista Anuário de Psicologia.

O índice de dispersão variou entre 1.97 para a contagem direta, 1.93 para a contagem completa e 2.15 para a contagem fracionada, indicando que a dispersão desta literatura é muito pequena e que os autores trabalham de forma isolada, resultando numa produção em parceria reduzida. Este resultado também é similar ao reportado por Rogge (1976), na antropologia Americana, na qual observou que somente $20 \%$ dos artigos publicados no The American Anthropologist e 30\% no American Antiquity eram trabalhos em parceria.

Artigo submetido em 18/02/2009 e aceito em 21/07/2009.

\section{REFERÊNCIAS}

ALLISON, Paul D. et al. Lotka's Law: a problem in its interpretation and application. Social studies of science, v. 6, n. 2, p. 269-276, 1976.

BAKER, Joseph; ROBERTSON-WILSON, Jennifer; SEDGWICK, Whitney. Publishing productivity in sport psychology, 1970-2000: an exploratory examination of the Lotka-Price Law. Journal of Sport \& Exercise Psychology, v. 25, n. 4, p. 477-483, Dec. 2003.

BERG, Jan; WAGNER-DÖBLER, Roland. A multidimensional analysis of scientific dynamics: part I: case studies on mathematical logic in the 20th century. Scientometrics, v. 35, n. 3, p. 321-346, 1996.

BOURDIEU, Pierre. O campo científico. In: . Sociologia. São Paulo: Ática, 1973, p. 122-155.

CARPINTERO, Helio; PEIRÓ, José María; QUINTANILLA, Ismael. El "Anuario de Psicología” (1969-1974): un estudio estadístico y bibliométrico. Anuario de Psicología, v. 16, n. 1, p. 22-34, 1977.

CATTELL, J. Mckeen. A further statistical study of American men of science. Science, v. 32, p. 633-648, Friday, Nov. 1910.

CHUNG, Kee H.; COX, Raymond A. K. Patterns of productivity in the finance literature: a study of bibliometric distributions. The Journal of Finance, v. 45, n. 1, p. 301-309, Mar. 1990.

COILE, Russell C. A bibliometric examination of the square root theory of scientific publication productivity. In: FRY, Bernard M.; SHEPHERD, Clayton A. (Ed.). Information management in the 1980s: proceedings of the 40th ASIS Annual Meeting. White Plains, New York: American Society for Information Science, 1977. Volume 14, Chicago, Illinois, September 26-October 1, 1977: Part 1: Abstracts of papers; Part 2: Full papers.

A bibliometric examination of the square root theory of scientific publication productivity. Arlington, VA: Center for Naval Analyses, 1977. 6 leaves. (Professional paper / Center for Naval Analyses; n. 205).

DENNIS, Wayne. Variations in productivity among creative workers. The Scientific Monthly, v. 80, n. 4, p. 277-278, Apr. 1955.

DRESDEN, A. A report on the scientific work of the Chicago section, 1897-1922. Bulletin of the American Mathematical Society, n. 28, p. 303-307, July 1922.

GLÄNZEL, W.; SCHUBERT, András. Price distribution: an exact formulation of Price's "Square root law". Scientometrics, v. 7, n. 3-6, p. 211-219, Mar. 1985.

GUPTA, B. M.; KARISIDDAPPA, C. R. Author productivity patterns in theoretical populations genetics, 1900-1980. Scientometrics, v. 36, n. 1, p. 19-41, May 1996. 
; et al. Distribution of productivity among authors in potato research (1900-1980). Library Science with a Slant to Documentation, v. 33, n. 3, p. 127-134, Sept. 1996.

; SHARMA, Lalita; KUMAR, Suresh. Literature growth and author productivity in Indian physics. Information Processing and Management, v. 34, n. 1, p. 121-131, 1998.

LOLAS, Fernando. Sobre la medición de la ciencia. Vida Médica, Santiago, v. 37, n. 2, p. 42-46, 1986.

McCREERY, Laurie S.; PAO, Miranda Lee. Bibliometric analysis of ethnomusicology. In: FLOOD, Barbara; WITIAK, Joanne; HOGAN, Thomas (Comp.) Challenges to an information society. ASIS ANNUAL MEETING, 47., 1984, New york. Proceedings... New York: Knowledge Industry Publications, 1984. p. 212-216.

MOLES, Abraham A. Theorie de linformation etperception esthetique. Paris: Flammarion, 1958.

Information theory and esthetic perception. Urbana, Illinois: University of Illinois Press, 1966.

NAGPAL, O. S. et al. Trends of researchin electronics enginnering: an analysis of publications by indian authors. New Delhi: Council of Scientific \& Industrial Research, 1966.

NICHOLLS, Paul Travis. Price's square root law: empirical validity and relation to Lotka's Law. Information Processing and Management, v. 24, n. 4, p. 469-477, 1988.

Examining fundamentals laws and methods of bibliometrics. Canadian Library Journal, v. 45, p. 247-248, August 1988.

NIJAGUNAPPA, R.; GUNJAL, S. R.; PARVATHAMMA, N. Indian Earth Science Literature (1978-88): a bibliometric study. In: GUPTA, B. M. (Ed.). et al. Handbook of libraries, archives and information centres in India. New Delhi, India: Information Industry Publications, 1996. Bibliometrics, Scientometrics and Informetrics, v. 13, p. 237-247.

PRICE, John Derek de Solla. Litle science, big science. New York: Columbia University Press, 1963.

Litle science, big science... and beyond. New York: Columbia University Press, 1986.

The productivity of research cientists. In:ENCYCLOPAEDIA Britannica: yearbook of science and the future. Chicago: Encyclopaedia Britannica, Inc., 1975. p. 409-421.
Galton revisited. In: Litle science, big science. New York: Columbia University Press, 1963. p. 33-61.

Networks of scientific papers. Science, v. 149, n. 3683, p. $510-515,1965$

Some remarks on elitism in information and the invisible college phenomenon in science. Journal of the American Society for Information Science, v. 22, n. 2, p. 74-75, 1971.

The structure of publication in science and technology. In: GRUBER, William H.; MARQUIS, Donald G. (Ed.). MIT Conference on the Human Factor in the Transfer of Technology, 1966, Endicott House. Factors in the transfer of technology. Cambridge, MIT Press, [1969]. p. 91-104.

RAHMAN, Abdur.; MALIK, S. Current trends of research in botany in India. New Delhi: Research Survey and Planning Organization, 1966.

RIVAS, Francisco; PEIRO, José María. Estado actual de la investigación psicométrica: una aproximación bibliométrica. In: CARPINTERO, Helio et al. Psicología contemporanea: teoria y metodos cuantitativos para el estudio de su literatura científica. Valencia: Alfaplus, 1981. p. 199-218.

ROGGE, A. E. A look at academic anthropology: thorugh a graph darkly. American Anthropologist, v. 78, n. 4, p. 829-843, Dec. 1976.

URBIZAGÁSTEGUI ALVARADO, Rubén. Lotka's law: an annotated bibliography, Riverside, CA: 2005. Unpublished.

Pierre Bourdieu: a bibliography. Riverside, CA: Waira Publications, 1993.

; CORTÉS, Maria Teresa. Método gráfico para medir la obsolescencia de la literatura geologia: el caso de la Revista Geológica de Chile. Investigación Bibliotecológica, México, v. 24, n. 12, p. 81-98, enero/jun. 1998.

VLACHY, Jan. Variable factor in scientific communities: observations on Lotka's law. Teorie a Metoda, v. 6, n. 1, p. 91-120, 1972.

Distribution patterns in creative communities. In: WORLD CONGRESS OF SOCIOLOGY, 8., 1974. Toronto. 